and fruitful channels. If we know the modifications in function arising from physical or chemical changes in the environment of the functionating cell, we at once obtain means of controlling such function more or less, and what this means to the physician who has to deal with manifestations of disturbed function in his patients needs no amplification. In this way he frequently may obtain the desired effect in spite of the fact that the ultimate mechanism of the action is unknown, and a complete knowledge of the same possibly unattainable.

Such studies of function under carefully selected and well-controlled experimental conditions may also permit of very interesting conclusions with regard to the mechanism involved. Our knowlcdge of the chemical control of glandular function has been obtained in this way. There is also no question that some serious errors arising from a merely anatomic study of the parts involved have been corrected by this method, but after all it is a method with its own advantages and its own palpable limitations like all others.

In conclusion, then, I wish to emphasize that our goal of a thorough understanding of the biologic processes in health and disease cannot be reached by one method alone. Men with varying inclinations and varying training have to cooperate in order to make progress, and the more intricate our problems grow the more the necessity of such cooperation becomes evident.

It is of course an impossibility for any man to be a master in all branches of biologic research, but a fair degree of familiarity with all is necessary, to produce, if I may borrow a German expression, a friendly understanding, to avoid misconceptions and the development of that narrowness of view to which I imagine Professor Henderson wished to refer. I believe that laboratories in pathology, just like those in physiology, pharmacology, hygiene and clinical medicine, should be ready at any time to take up a problem from whichever point of view appears to be the most hopeful, with whatever methods seem to be indicated, and, if necessary, from as many points of view and with as many variations in method as seem desirable.

\section{THE PRESENT STATUS OF PYELOGRAPHY *}

\section{FLOYD E. KEENE, M.D.}

HENRY K. PANCOAST, M.D. PHILADELPHIA

Sufficient evidence is furnished by the excellent work of Braasch and many others to establish without question of douht the value of pyelography in the diagnosis of surgical diseases of the kidney and ureter. Additional testimony based on our own experience would mean merely a repetition of what has already been stated. Another phase of the subject, which is of equal importance and forms the theme of this paper, is the consideration of the harmful results that may follow collargol injection, its indications and limitations.

When we first began the use of pyelography, we shared the opinion of others that the injection of

* Read before the Section on Obstetrics, Gynecology and Abdominal S.rrgery at the Sixty-Fifth Annual Session of the American Medical Association, Atlantic City, N. J., June, 1914. collargol into the renal pelvis was free from danger and employed it routinely in all cases presenting symptoms which might be due to renal pathologic conditions. In the course of time we became convinced of the error of such a procedure. With increasing knowledge acquired from our own experience as well as that of others, we have put a decided limitation on the cases suitable for injection and have formulated a technic which we believe will reduce the disastrous sequelae of pyelography to the minimum.

That collargol produces little or no irritation of the pelvic mucosa seems to be proved by clinical and experimental evidence. Following injection in twenty of our cases, the urine rapidly lost the collargol stain, and microscopic examination was normal or showed only a slight excess of leukocytes with an occasional erythrocyte. Kidd ${ }^{1}$ reports similar results. In his discussion of Kidd's paper, Walker states that he "has operated on a number of cases within a few days of the collargol examination and in every case the lining membrane of the renal pelvis was healthy and glistening even when the pelvic contents were deeply stained

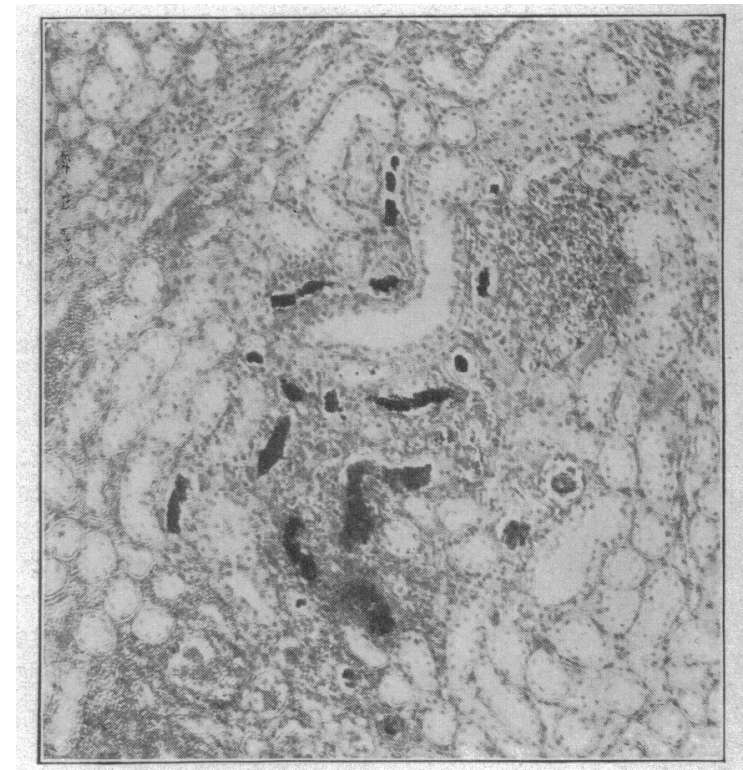

Fig. 1.-Early tuberculosis of the kidney. From photomicrograph. Intense infiltration of collargol throughout all portions of the section
with well-marked destruction of kidney structure. High pressure used in making the injection.

with collargol. In some cases where nephrectomy was performed the mucous membrane was normal microscopically." This coincides with our own findings; in both specimens illustrated in this paper, the lining epithelium of the pelvis is normal although the kidney shows wide-spread changes. Blum, ${ }^{2}$ on the other hand, reports extensive necrosis, but, as Strassmann ${ }^{3}$ points out, his experiments are open to criticism in that he used post-mortem specimens, and the changes which he ascribes to collargol could well have been cadaveric. The burden of proof, therefore, is that, so far as the renal pelvis is concerned, collargol is harmless.

That the injection may have a decidedly injurious effect on the kidney has been shown repeatedly and almost without exception; the pathologic reports detail the same results, namely, the tubules filled with collargol, which may extend into Bowman's capsules, rup-

1. Kidd, Frank: Proc. Roy. Soc. Med., 1913, vii.

2. Blum: Wien. med. Wchnschr., 1912, No. 19

3. Strassmann: Ztschr. f. urol. Chir., March, 1913, p. 126. 
ture of the tubules, peritubular round-cell infiltration, renal infarcts or areas of cortical necrosis. Strassmann alone maintains that the tubules are free, the collargol being taken up by the renal lymphatics or tissue clefts. The experimental work of Tennant, ${ }^{4}$ Oehlecker, ${ }^{5}$ Kidd and others, conclusively proves that collargol gains entrance to the kidney by way of the tubules and that the extent of involvement is directly dependent on the degree of pressure exerted in making

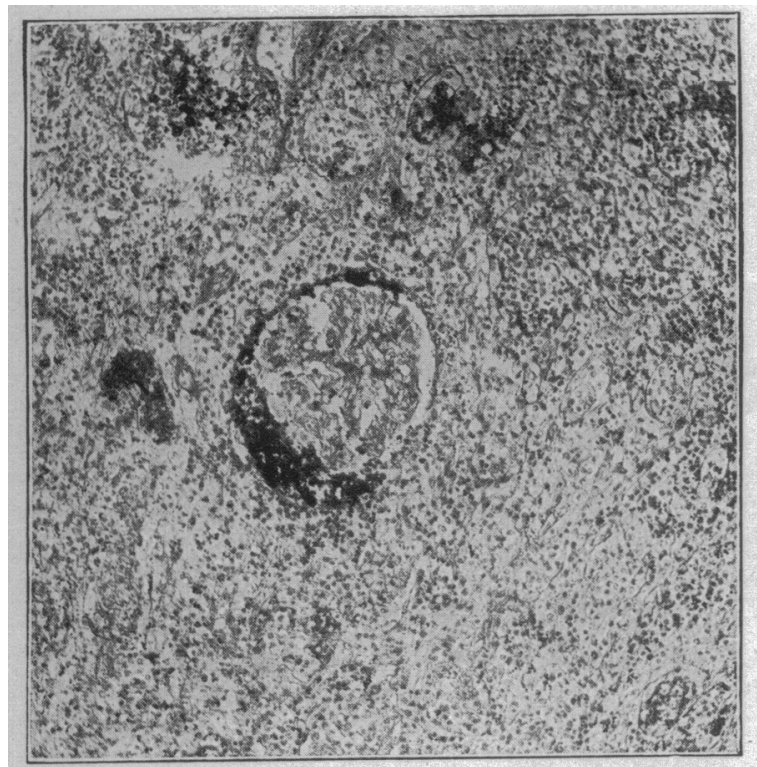

Fig. 2.-Same specimen as shown in Fig. 1. From photomicrograph Argyrol in Bowman's capsule, also permeating the kidney tissue with a moderate degree of necrosis and round-cell infiltration.

the injection as well as the time that the solution is allowed to remain in the pelvis. The same results are reported clinically by Mason, ${ }^{6}$ Tennant $^{4}$ and Troell. ${ }^{7}$ The accompanying photomicrographs (Figs. 1, 2 and 3 ) depict similar conditions. These injections were made during our earliest experience in pyelography and represent the ill results that may attend imperfect technic in types of disease in which, if used at all, the injection must be made with the greatest skill and care. Severe pain followed both injections and confirms Tennant in his statement that pain following collargol injection may have a graver significance than the mere overdistention of the pelvis, indicating the passage of the solution into the renal parenchyma.

The question naturally arises, Is the kidney permanently or seriously injured by collargol in the absence of superadded infection or such extensive lesions as infarction or necrosis? Pathologic evidence and clinical experience seem to indicate that this can be answered in the negative. Leukocytes, a few red blood-cells and hyaline casts may be present immediately following injection, but these soon disappear. Even in those cases in which violent pain and high fever have followed, such as reported by $\mathrm{Vest}^{8}$ and Zachrisson, ${ }^{9}$ the urine sooner or later returns to normal.

4. Tennant: Ann. Surg., 1913, lvii, 888

5. Oehlecker, F.: Fortschr. a. d. Geb. d. Róntgenstrahlen, 1911 xvii, 195.

6. Mason, J. M.: Dangers Attending Injections of the Kidney Pelvis for Pyeiography, The Journal A. M. A., March 14, 1914, p. 839.

7. Troell: Hygiea, 1913,75, p. 176 ; ref. in Zentralbl. f. ges. Chir.

u. d. Grenzgeb., 1913, i, No. 16, p. 829 .

8. Vest: Bull. Johns Hopkins Hosp., March, 1914, p. 74.

9. Zachrisson, F.: A Case of Collargol-Injection into the Tubuli Recti of the Kidney, Nord. Med. Ark., 1911, xi, ref. in Zentralbl. $f$. Chir., 1911, No. 50 .
Evidence is available, therefore, which shows that wide-spread lesions of the kidney may result from collargol injection and as a rule go hand in hand with overdistention of the renal pelvis. In many cases, collargol may find its way into the straight tubules, but the resulting irritation of the renal parenchyma is usually trifling and produces no permanent injury. That a substance may be found which will be nonirritating as well as impermeable to the Roentgen ray is to be hoped for. Kelly and Lewis ${ }^{10}$ advocate silver iodid but their report is too meager to warrant acceptance until further accurate experimental and clinical evidence is submitted.

Our experience, together with a study of the cases reported in literature, convinces us that too much stress has been put on the supposed corrosive action of collargol, while in reality the fault lies in the method of injection or its application in types of disease which favor rapid permeation of the renal tissue.

As the result of his experiments with Berlin blue and other inert anilin dyes, Marcus ${ }^{11}$ demonstrates with what ease trauma to the pelvis and kidney may be produced by even low pressure. If no injury were present, the coloring-matter merely lay in the pelvic clefts or ascended occasionally into the collecting tubules at the papillae, but was never found in the intratubular tissue. When the dye was found in the veins of the kidney, he invariably demonstrated a traumatic site of entrance in the pelvis or renal parenchyma. Under high pressure, it was an easy matter to produce a migration of any inert substance from the pelvis to the renal vein by way of the kidney, with emboli in various organs. He also shows, experimentally, that the site of rupture determines the area per-

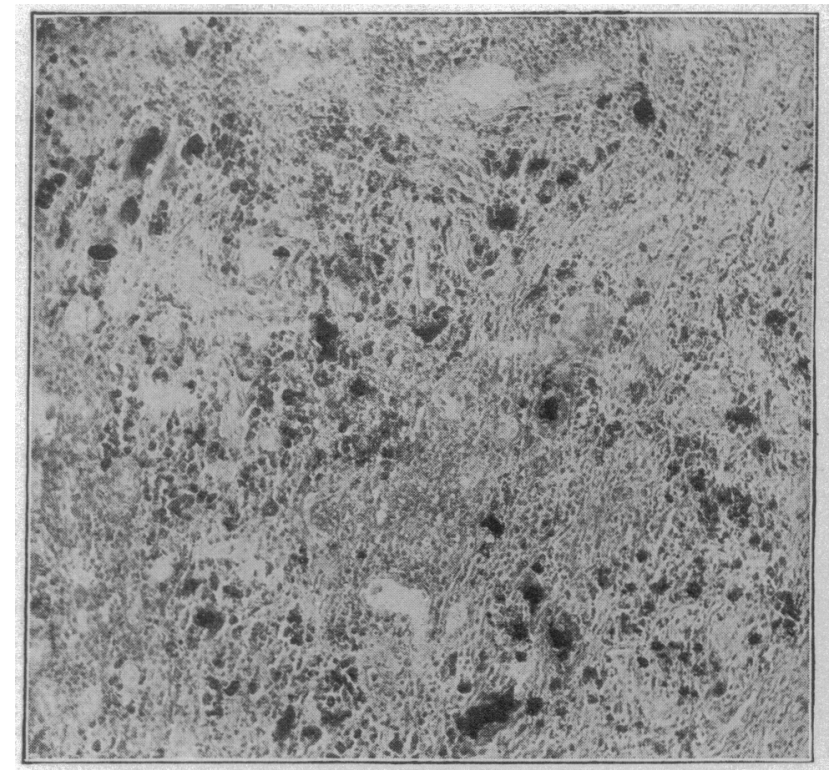

Fig. 3.-Pyelonephritis and hyćronephrosis. From photomicrograph. Fifty c.c. of a 10 per cent. argyrol solution were injected under high pressure. In this section the argyrol is confined within the tubules; the kidney tissue is well preserved.

meated by the dye which may be found in the renal vein, the subcapsular space or the peripelvic tissue.

The experimental work of Lewin ${ }^{12}$ was done along similar lines and with like resilts, although he does not emphasize the importance of trauma as the exciting

10. Kelty and Lewis: Surg., Gynes, and Obst., 1913, xvi, 707.

12. Lewin: Arch. f. exper. Path. u. Pharmakol., 1897-98, x1, 287 
factor in permitting the escape of the injected fluid. Emboli were found in the right heart, lungs and liver, corresponding to the recent report of Eisendrath ${ }^{13}$ and differing only in the fact that he used an anilin dye instead of collargol. The experiments of Strassmann, Kidd and Tennant, previously referred to, substantiate the findings of these investigators and show the possi-

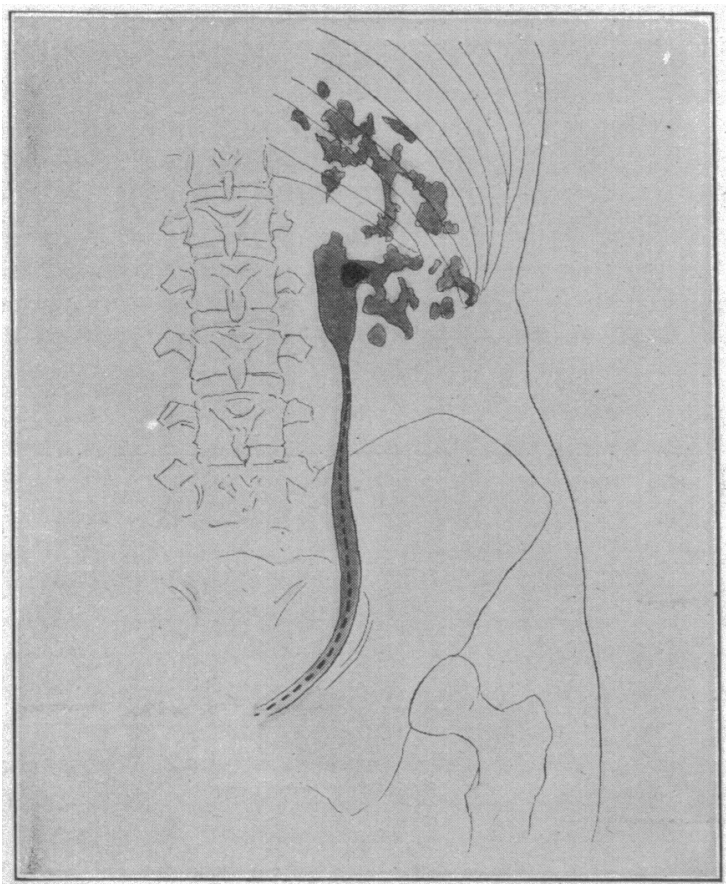

Fig. 4.-Calculus pyonephrosis showing diffuse permeation of collargol throughout kidney. Type of case unsuitable for pyelography.

bilities of danger which attends injection under high pressure, and also the relative freedom from danger when little or no distention of the pelvis is produced.

It is doubtless true that, in the earlier days of pyelography, many instances of severe pain or disastrous results following injection were due to an erroneous conception concerning the capacity of a normal pelvis. At that time the limits were stated by various observers as varying from 15 to 30 c.c., while at present we know that the capacity is between 2 and 5 c.c. It is only natural to assume, therefore, that, in making the injection, the normal capacity was many times exceeded.

We are firmly convinced that solution of continuity of tissue, whether by ulceration, or direct or indirect trauma, plays an important rôle in permitting the escape of collargol from the pelvis. The work of Marcus already referred to shows how even low grades of distention can break the pelvic lining in normal kidneys. In kidneys altered by disease, whether this be a thinning out as in hydronephrosis, an ulceration as in tuberculosis or tumor, or increased friability due to pyogenic infection, it is self-evident that ruptures of varying degrees are more prone to result. Of the reports in literature of cases in which extensive changes have been produced by collargol, it is interesting to note three cases of hypernephroma (Mason, Oehlecker and Rössle $\left.{ }^{14}\right)$, one case of pyonephrosis

13. Eisendrath, Daniel: The Effect of Injecting Collargol into the Tenal Pelvis, The Journal A. M. A., May 2, 1914, p. 1392.

14. Rössle: Tödliche Kollargolvergiftung, München. med. Wchnschr., 14. Rössle: T
1911, lviii, 280.
(Troell) and one case of suppurative pyelonephritis (Schwartzwald ${ }^{15}$ ).

To these we add the cases herein reported, one of early tuberculosis and the other chronic pyelonephritis and hydronephrosis (Fig. 4). This is sufficient evidence to indicate that such lesions may act as at least contributing factors in the dissemination of collargol throughout the kidney, since they furnish ideal conditions for the production of a portal of entrance.

The effect of direct trauma by the urreteral catheter is nicely shown in the specimen of Mallory reported by Mason: "The collargol was injected into the kidney tissue as a result of running the catheter too far into the pelvis of the kidney: Evidently the point of the catheter went into a calyx and the fluid was injected under pressure into that calyx." Any one experienced in ureteral catheterization knows that unless the greatest care is used, it is an easy matter to traumatize the pelvis sufficiently to produce bleeding and thus bring about a condition similar to that seen in the specimen just mentioned.

Reviewing the cases reported in literature from the clinical point of view, pain following injection is the most frequent symptom. This may be very severe, requiring morphin for its relief, and persists for several hours or even days. Pain may follow the most careful manipulation in passing the catheter or the injection of only a few drops of collargol. In our. experience this has occurred only rarely; when severe pain has resulted, it has been due to our faulty technic of catheterization or injection. When pyelography was first undertaken, we were inclined to ascribe this

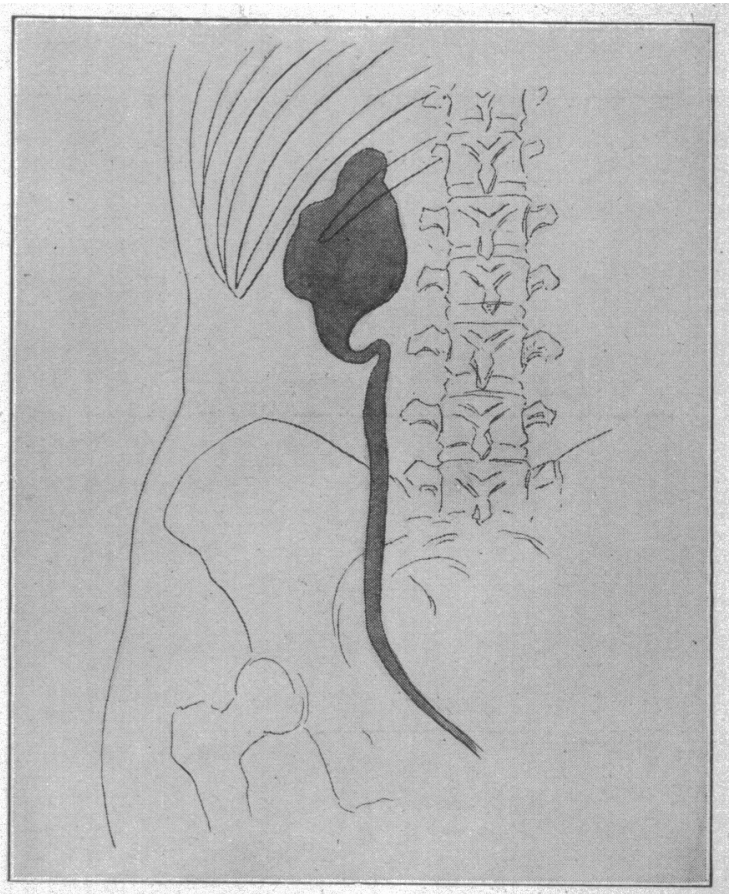

Fig. 5.- Colon bacillus pyelitis with marked dilatation of pelvis due to ureteral kink. Symptoms relieved by pelvic lavage. The usual methods
of treatment together with autogenous vaccines had been unsuccessful.

pain to the irritation of the collargol, and varied the percentage of the solution from 1 to 10 , with no apparent relationship between the strength of the solution and the intensity or absence of pain. It was evident that some other factor was active. We found

15. Schwartzwald: Beitr. z. klin. Chir., lxxxviii, No. 2 
that the frequency of pain was greatly diminished by inserting the catheter only part way up the ureter instead of into the pelvis. This plan is open to criticism in that it gives no idea of the pelvic capacity, but it does indicate that the avoidance of trauma to the pelvic mucosa is an important factor in lessening the harmful results, of which pain is often the only

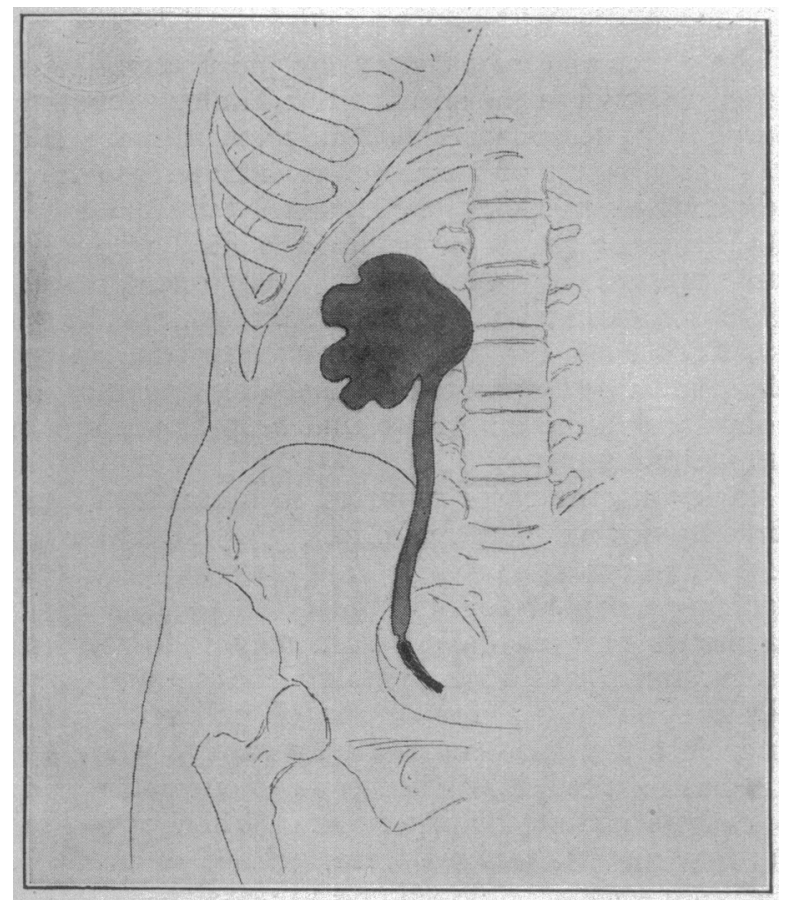

Fig. 6.-Stricture of ureter. Marked dilatation of ureter above stricture, and pyelonephritis of gonococcal origin. Pyelography was employed in order to eliminate cdlculus as a possible cause of ureteral obstruction. Lavage and collargol instillation resulted in temporary improvement.

symptom. As further proof of the ill effects of trauma, we have observed that pain almost constantly accompanies injection in cases in which bleeding follows catheterization.

The second and by far the most important cause of pain is overdistention of the renal pelvis. Whether we accept the evidence of Marcus that overdistention produces solution of continuity of the pelvic lining, or of other observers that the collargol ascends directly into the straight tubules without previous pelvic injury, the fact remains that, as a rule, the degree of permeation in the kidney is in direct relationship to the amount of pressure exerted by the distending medium.

We are in full accord with Tennant's statement that pain indicates in many instances the passage of collargol into the kidney tubules, and is not merely a colic incident to an overfilled pelvis. If the latter were true. the pain should cease shortly after the relief of pressure rather than persist for hours or days. We ascribe it to increased tension of the renal capsule, produced primarily by the sudden increase in volume of the kidney due to the entrance of collargol, and secondarily to reactive changes within the kidney incident to the injection of a mildly irritating, foreign substance. In seventy cases in which the piston syringe was employed, pain resulted in over 10 per cent.; in our last thirty cases, we have used the gravity method of injection as recommended by Thomas ${ }^{16}$ with no

16. Thomas, G. J.: An Apparatus for the Injection and Lavage of
the Pelves and the "reters, ThE Journal A. M. A., Jan. 18, 1913, p. 184 . pain whatever, proving conclusively its great advantage over the older method.

Several cases are reported in which fever has followed injection, persisting for days and in a few instances attended by the development of perirenal suppuration. As first pointed out by Voelcher and observed by Braasch and many others, collargol is not uncommonly found in the peripelvic tissue and produces little or no inflammatory reaction. We are convinced that a rise in temperature is due not to collargol per se, but that faulty technic has permitted the introduction of infection, with collargol as the medium by which it is carried to the kidney or its surrounding tissues.

Lastly, three deaths are reported as occurring after collargol injection. The often-quoted case of Rössle has been criticized by Strassmann who advances arguments which tend to disprove that collargol was the cause of death. More recently a second case is reported by Rosenblatt and Morgandies, ${ }^{17}$ in which a state of shock developed at the time of injection with death several hours later. In this case of suspected hydronephrosis, 40 c.c. of a 5 per cent. collargol solution was injected without difficulty. A post-mortem examination was not performed nor was the kidney examined microscopically. The perirenal tissues were widely infiltrated with collargol, there was no collargol in the pelvis or ureter and only a "minimal" tear was visible in the kidney tissue. In a case reported by Vest, death resulted on the fourteenth day after operation. Acidosis and acute dilatation of the stomach were postoperative complications. Vest does not

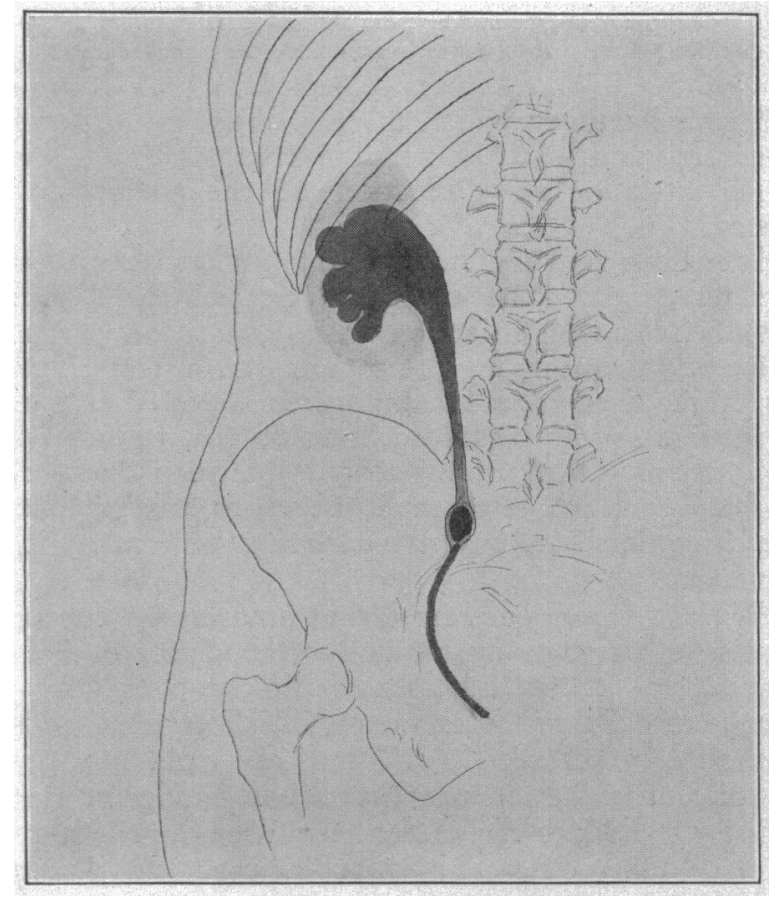

Fig. 7.-The roentgenogram failed to demonstrate a calculus. The catheter met an obstruction $13 \mathrm{~cm}$. from the ureteral orifice. By means of a collargol injection, the calculus was clearly defined, together with a hydro-ureter and hydronephrosis.

assert that death was due to collargol poisoning, but draws attention to the interesting fact that, as in Rössle's case, hemorrhagic diathesis developed a few days before death. In his preliminary report, Eisen-

17. Rosenblatt and Morgandies: Verhandl. d. deutsch. Röntg. 17. Rosenblatt and
Gesellsch., 1913, ix, 81. 
drath has shown experimentally that death may result from emboli in various organs, but this has occurred only when collargol was injected under very high pressure. That similar results may follow injection in man is of course true, but with ordinary care and skill, we can state without fear of contradiction that this danger can be entirely eliminated.

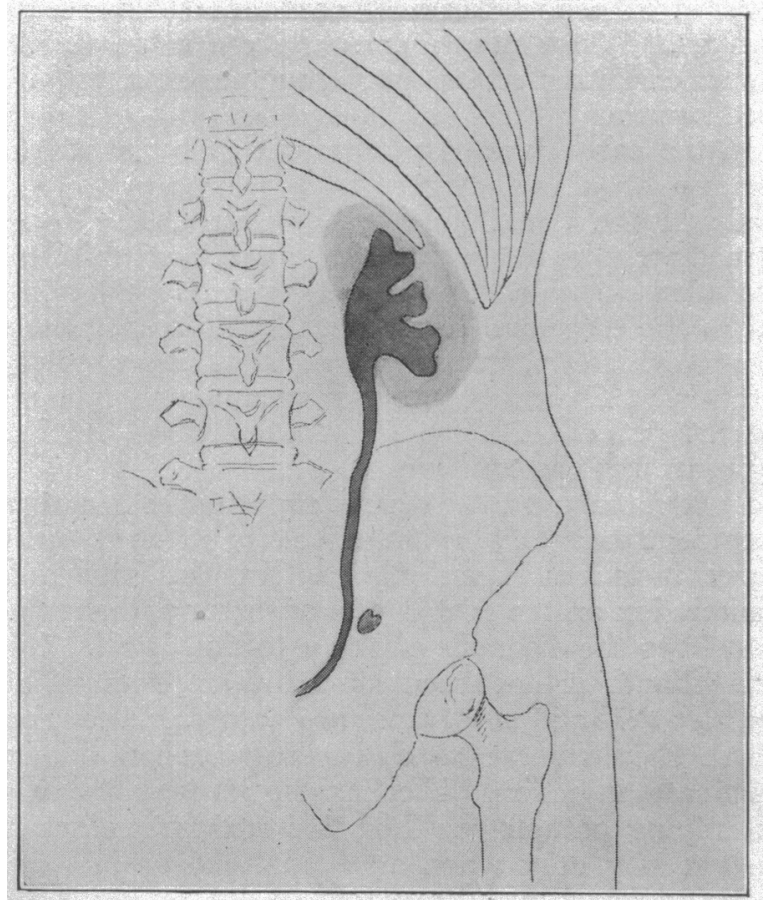

Fig. 8.- History in this case indicated ureteral calculus. A small cystic mass lay to the side of the uterus and the roentgenogram showed a shadow along the course of the ureter. Pyelography demonstrated a normal pelvis and ureter and an extra-ureteral shadow, which operation proved to be a tooth in a dermoid cyst.

\section{CYSTCSCOPIC 'TECIINIC}

Basing our ideas on experimental evidence as well as clinical experience, we have formulated certain points in technic which, in our opinion, are of importance in avoiding the untoward results of collargol injection. The greatest care as regards asepsis cannot be too strongly emphasized. The ureteral catheter should not exceed No. 6 in size, it must be free from rough surfaces and pliable so as easily to follow any change in the course of the ureter; to avoid trauma, we never use a stiletted catheter. The catheter is inserted for a distance of $20 \mathrm{~cm}$. and its further progress is made extremely slowly until the slightest buckling occurs, which indicates that its tip has reached the pelvis. It is then withdrawn 1 or $2 \mathrm{~cm}$. and the character of the urinary outflow is observed to determine, if possible, the presence or absence of pelvic dilatation. The catheter is then withdrawn $10 \mathrm{~cm}$. and the injection made. By so doing the danger of wounding the pelvis is minimized and the tip of the catheter is below the level at which the majority of ureteral kinks are found.

If the urine is blood-stained, the injection is not made, a subsequent examination being advised at an interval of at least seven days. When an obstruction is encountered along the ureter, we do not attempt forcibly to overcome it, but pass a smaller catheter; if its passage is likewise impeded, the collargol is injected and in the majority of instances, will find its way upward. The forcible passage of the ureteral catheter is to be condemned under any circumstance, but this is especially true when injection is to follow. We are opposed to the routine practice of simultaneous injection of both kidneys; when bilateral disease is suspected, the injections are made at two sittings. If for any reason, the roentgenogram is unsatisfactory, we make it a rule to allow several days to elapse before attempting a second injection.

The collargol is freshly prepared for each case; the crystals are thrown into a small mortar containing boiling water and when cooled are thoroughly dissolved by stirring with a pestle. The solution is filtered through cotton and gauze and is ready for injection. The strength of the solution varies from 5 to 10 per cent., depending on the thickness of the abdominal walls. In making the injection, we use a 30 c.c. buret connected with a short tube and stopcock. To start the flow through the catheter, elevation of the buret to about 3 feet will be necessary but it is immediately lowered and allowed to flow in at an elevation of not more than 1 foot with a No. 6 , and 2 feet with a No. 5 catheter. The injection is discontinued when the column of collargol ceases to fall or the patient experiences the first slight sensation of fullness in the kidney region. After the picture is taken, the collargol is drained off and the catheter removed.

We make it an invariable rule to keep our patients under observation in the hospital for twenty-four hours after injection and prescribe copious draughts of water during the first six hours. When retention from

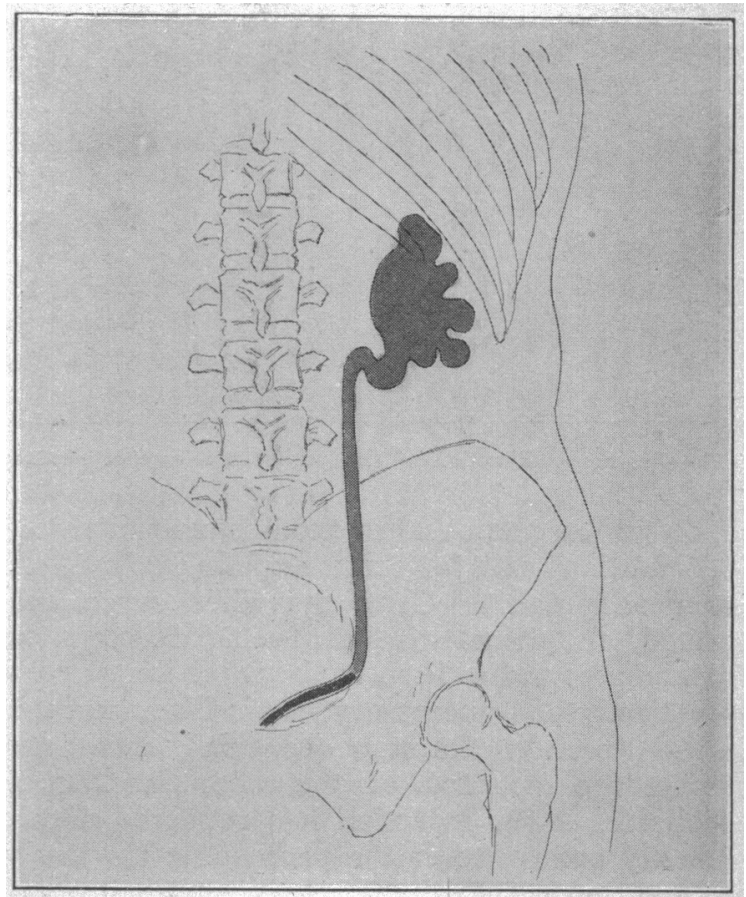

Fig. 9.- Ptosis of the left kidney with hydronephrosis. The catheter met an obstruction $4 \mathrm{~cm}$. from the ureteral orifice. Pyelography demonstrated that the obstruction was not pathologic, but due to an abrupt change in the course of the normal ureter.

angulation of the ureter due to ptosis is suspected, the patient is required to remain in bed for twelve hours after the injection. This facilitates free drainage of any collargol that may remain in the pelvis of the kidney. 


\section{ROENTGENOGRAPHIC TECHNIC}

The diagnosis of renal and ureteral conditions by pyelography depends on the correct interpretation of the roentgenogram made immediately after a careful ureteral catheterization and injection with the opaque solution. While each step is of equal importance, and each without the other is of little or no value, roentgenography plays but a minor part in the procedure as a whole. It is to be regarded simply as a means of accurately demonstrating and recording what the cystoscopist has done. The real value of the method arises in a very large measure from the technic of the cystoscopist in creating correct appearances for the roentgenogram to portray in order to demonstrate existing conditions accurately.

When employed as a safe and necessary procedure, pyelography has a very limited range of application as compared to the usual routine roentgenoscopy of the urinary tract. It is to be employed, as a rule, there-

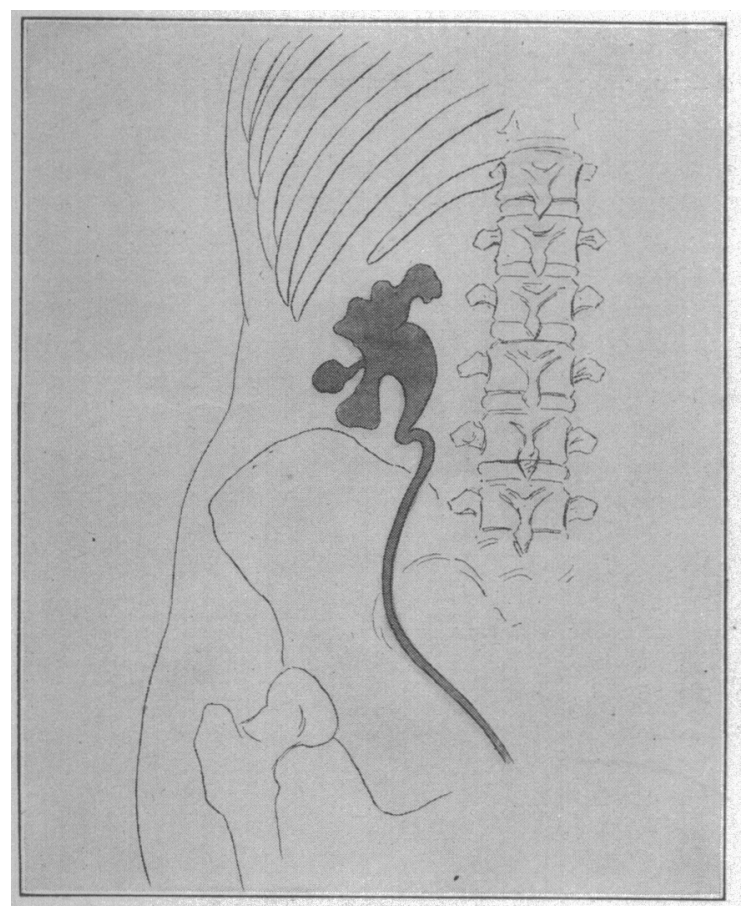

Fig. 10.-Beginning dilatation of the pelvis in a case of nephroptosis.

fore, only after the usual methods have failed to show any condition satisfactorily explaining the clinical phenomena present. Certainly, whenever calculus is suspected, the usual routine roentgenoscopy should always be resorted to first, as the injection of collargol may not only be unnecessary but is likely to interfere with the detection of a stone, especially in the kidney. Instances are recorded, on the other hand, in which the colloidal silver injection has rendered a calculus sufficiently opaque to cast a perceptible shadow when it was not detected by the simple roentgenoscopy alone. We are able to cite one experience of this kind in connection with a ureteral stone which was distinctly shown by the collargol injection,-. whereas no shadow could be detected in roentgenograms made at previous and subsequent examinations in the usual manner. Of course, such instances are excedtional.

There is little to be said of the roentgenographic technic per se. Preliminary preparation of the patient is always essential, and comprises the withholding of food, pills and tablets, and the thorough evacuation of the intestinal tract. As we have found castor-oil disagreeable and not always satisfactory in removal of gas, we have recently adopted the use of compound cathastic pills pulverized and administered in capsules, and have found this procedure more efficacious.

Compression, which is so essential in the usual roentgenoscopy of the urinary tract, had best be avoided in connection with pyelography, for obvious reasons. When stout individuals are to be examined, the intensifying screen may become essential, if not used routinely.

In the early days of pyelography, Stover advocated the use of a table whereby one exposure could be made in the usual horizontal posture, and repeated immediately in the semirecumbent or vertical position by tilting the table. Such a procedure is obviously useful in the diagnosis of ureteral kinks associated with movable kidneys, but we have found it by no means essential. The substitute performance of having the patient get off the table and stand for the second exposure is to be condemned.

In our analysis of the cases reported in literature, it is evident that the harmful effects of collargol have, in many instances, been due to sins of omission or commission on the part of the operator and should not be held to the discredit of the injection itself. On the other hand, it has been shown that, even with the greatest skill and care, there are possibilities of danger which should serve as a warning against the indiscriminate injection of collargol, irrespective of the inderlying pathology. For this reason, we resort to pyelography only when an accurate diagnosis cannot be made by other methods of examination.

We are opposed to its use in depicting interesting anomalies when such a procedure means merely a graphic representation of what may be accomplished by the method. We are likewise opposed to its indiscriminate use in all types of renal pathology; for experimental as well as clinical evidence indicates that possibilities of harm are certainly increased in the presence of ulceration, infection or retention of the solution in the pelvis. For this reason, with only rare exceptions is the injection made in suspected cases of neoplasm, tuberculosis or large hydronephrosis. Likewise, in those infections other than tuberculosis, we hesitate to employ collargol until other methods of diagnosis have been exhausted. It is of the utmost importance in the treatment of pyelitis to determine whether we are dealing with a mechanical dilatation of the pelvis with superimposed infection or with a true primary infectious pyelitis, and if other methods fail to reveal the desired information, the risk of pyelography is far outweighed by the accurate data which it affords.

When renal or ureteral calculi can be definitely recognized in the roentgenogram, little or no additional information will be afforded by pyelography; the injection is indicated only when the position and character of the shadow make it impossible to determine whether it lies within the kidney or ureter or in adjacent structures. This applies especially to those cases in which in addition to the shadow along the course of the lower ureter, the catheter meets with an obstruction at a corresponding level. Such evidence is of course strongly in favor of calculus, but is by no means positive, since inability to pass the catheter above a certain level is not infrequently due to an 
abrupt change in the course of the normal ureter. This point will be quickly determined by the presence or absence of ureteral dilatation above the site of obstraction.

The pyelograph serves its greatest field of usefulness in detecting the earlier stages of hydronephrosis due to mechanical blockage of the ureter other than that caused by stone. In fact, it is the only means at our command which permits the diagncsis to be made without question of doubt. This apolies likewise to certain congenital anomalies, especially the horseshoe and the dystopic kidney. Under these circumstances, if proper technic be employed, collargol injection can be made with but the barest possibility of harmful sequelae.

That exceptions to these general rules in the selection of cases will aris a from time to time, is quite obvious, but such exceptions are rare, and in no way detract iron the general principles which we have formulated for our own guidance. We may err on the side of conservatism, but we are satisfied that it is likewise on the side of safety, for we are convinced that possibilities of danger exist even under the most ideal circumstances. In the majority of instances, however, disastrous results following collargol injection have been due not to the use but to the abuse of the method, and we are confident that if greater care be given to the technic of injection as well as to the selection of cases, the dangers of pyelography will be of minor importance as compared to the valuable information which its use will afford.

116 South Nineteenth Street.

\section{ABSTRACT OF DISCUSSION}

Dr. W. F. BraAsch, Rochester, Minn.: Undoubtedly Drs. Keene and Pancoast should be given great credit for calling our attention to the possibility of ill results from the use of pyelography. If we remember the history of cystoscopy we shall recall that illness and death were reported, so that for a while the method fell into discredit. Ureteral catheterization also was criticised so severely that many would not permit patients to be so treated. While undoubtedly its abuse may cause harmful results, nevertheless it will remain as an invaluable method of diagnosis in conditions in the ureteral tract. It is a peculiar thing that of the deaths recently reported as the result of pyelography, the cases have been almost entirely in the hands of men of little experience. Our experience in 2,000 cases treated with pyelography has not given us serious results which could be ascribed to its use in any case. In my early use of the method I have observed three cases of cortical lesion in which I should not have used it. I have laid down the fundamental rule never to make more exploration of the tract than is necessary ior diagnosis. There is a long list of conditions in which I have diagnosed obscure symptomatology which could not have been diagnosed in any other way. Our endeavor should be to find some substance which is harmless and which can be injected without causing the colloidal poisoning. If drainage is interfered with there will be trotble; if the kidney is overdistended there wil be trouble. By means of the gravity method we have overcome the dangers of overdistention, but we cannot always overcome the dangers of overretention, but the dangers are reduced to a minimum. The silver iodid advised by Kelly seems to me to be a valuable adjunct. It cannot be employed in the gravity method, but must be employed with the syringe because of its viscidity. The ideal substance should be sufficiently liquid to be used in the gravity method and one which would not cause necrosis if retained. Pyelography should be in the hands only of men with wide experience in the work.
Dr. Henry Dawson Furniss, New York: There are still many dangers in pyelography, due to the mechanical insult of its performance and to the irritating effect and toxicity of the substances used. The ideal substance must be nonirritating, non-toxic and soluble in both water and the blood. Eisendrath has done some good experimental work in the injection of argyrol and collargol under pressure, and has found these substances not only in the tubules of the kidney, but also in the interstitial connective tissue, as well as in other organs of the body. The wide distribution he found due to entrance of the collargol through ruptured veins. In the Genito-Urinary Section were reported four or five fatalities following the use of collargol.. In some, death was within a short time and in others followed within ten days or two weeks. In these late fatalities a hemorrhagic condition developed, with bleeding from the mouth, nose, stomach and under the skin; there were superficial ulcers in the mouth. In making pyelographs, my technic is to fill the bladder with boracic acid solution, put the patient on the table, have the Roentgen-ray plate and tube adjusted, then catheterize the ureter using a soft No. 5 or 6 catheter, clamp the cystoscope in position with a holder, and then make the injection under gravity. This saves a lot of unnecessary moving of the patient, which is disagreeable and tends to displace the catheter. The soft catheter is used, as stiff ones are capable of injuring the kidney substance so that the collargol can enter the circulation of the kidney substance. By using small catheters there is less danger of harm, as the collargol can escape alongside of the catheter. Even with the most careful technic severe pain often attends making a pyelograph. I am of the opinion that the pain is due to other factors than the simple distention, as I have never noticed such long and severe pain in the cases where I have distended the renal pelvis for diagnosis according to the method of Kelly. I agree with Drs. Keene and Pancoast that pyelography is a most valuable diagnostic aid and one to be used only when other methods do not furnish us the necessary knowledge. It is especially useful in early hydronephrosis and easily passable strictures of the ureter.

Dr. D. N. Eisendrath, Chicago: I have had occasion to use pyelography a great deal but have felt that I was not justified in using it with the syringe, unless $I$ knew just where the danger-line was. In my first case the patient, who was 54 years old, died within half an hour after the injection. I made up my mind there must be some reason why the collargol in the urine caused trouble. We knew that the gravity method was apparently the safest and so we elevated a column of collargol $3 \frac{1}{3} 3$ feet above the level of the table and caused a pressure of $30 \mathrm{~mm}$., injecting a certain series of animals with 10 per cent. collargol, about 8 ounces, and killed them at different intervals. We were the first to use the manometer and by measurement were able to state the exact amount of pressure used to inject the collargol into the high renal pelvis. In this first series there were no changes; we could find no collargol except in one kidney. We had proved, experimentally, that the collargol was perfectly safe so long as we did not raise the pressure above $30 \mathrm{~mm}$. In the second series we increased our pressure from 30 to $70 \mathrm{~mm}$. and still kept as close as we could to the capacity of the renal pelvis of the $\operatorname{dog}-$ from 2 to $71 / 2$ c.c.-seidom over 5 c.c. The animal lived. There was collargol in the lungs, in small quantity in the liver and in the kidney. In the animals that lived for thirty days after the experiments, what Drs. Keene and Pancoast have said, was true; the collargol did not kill off the epithelium, but we got much inflammatory reaction. Then we decided to increase our pressure. The first animal in this series we subjected to a pressure of $100 \mathrm{~mm}$. and injected 20 c.c. and it died in five minutes from the time of beginning the injection. By the time the last of the fluid was in, the animal was dead. At necropsy we found that the lungs were perfectly black. They evidently had been filled completely with the collargol. The collargol filled every capillary of the lung as if injected for histologic purposes. The liver showed nodules, with quantities of collargol in the form of 
infarct deposits. It was evident that when the collatgol was injected it went up through and between the tubules and broke into the veins. We had to make almost a hundred sections before we could get one which showed the collargol breaking into the veins. In one specimen, with 20 per cent. collargol solution injected under considerable pressure, the collargol was shown actually breaking into a vein. The other high-pressure series showed that the animals which lived longer than a few minutes or half an hour had all the pulmonary symptoms and hemorrhages corresponding to the human cases from hemorrhagic infarcts or pneumonia. I helieve that the dangers lie entirely with the use of high pressure and the combination of the mechanical and toxic actions of the silver.

Dr. Arthur E. Hertzler, Kansas City, Mo.: The great toxicity of silver is just beginning to be recognized. Twelve years ago I published a paper on this suljject. Silver is very active in solution of $1: 10,000$. The manner in which it works is a matter of much controversy. It used to be said that a silver album:nate is not produced. It is astonishing how small an amount of carbon will produce a large amount of black substance. A single c.c. in a large-sized rabbit will stain a whole peritoneum and cause death. The capacity of the epithelium to prevent absorption is lost and the silver continues further down. The question is, How does the silver get to most parts of the body? I have made a large number of sections of all the organs of the body and sometimes found in the connective tissue bits of silver deposit. It looks as though the silver got there in solution. Yet from the general action of silver this seems impossible. When silver breaks into a vessel it attacks the leukocytes and these leukocytes travel to the distant organs. The question is, What kills the animals? Nowhere do deposits of silver seem to be enough for this.

Dr. John G. Clark, Philadelphia: This clinical method like many others, is open to criticism if it is done badly, but, after all, does not the same criticism apply to many valuable methods in vogue in other branches of medicine? If pyelography is practiced under such careful restrictions as are laid down by Drs. Keene and Pancoast, it can only he of great benefit and in no instance have I seen a single untoward result. In one case in which the ureter had been catheterized by Dr. Keene there was acute pain followed by a slight rise of temperature. I was under the impression at the time that a collargol injection had been made and was inclined to criticise the method. I found, however, that only a simple catheterization had been done and there could therefore be no reflection on this valualsle method which we are discussing. Subsequently, this same patient had a collargol injection and not the slightest reaction followed it. In gynecologic work, as was shown in a paper which Dr. Keene and I published last year, irritability of the hladder is a very common symptom; so common, indeed. that many times it is accepted without question as coming from some pelvic disability. Oftentimes a gynecologic operation is done which is not followed by relief and the patient is looked on as a neurasthenic. In many of these cases, a cystoscopic examination with a pyclographic study clears up the doubt at once and locates the difficulty either in the ureter or the kidney. A large number of such cases have come under our care and I have the very highest regard for this work and feel that it has a most important place in our diagnostic methods. Even if we assume there is occasional danger, the method is so valuable in the aggregate that I do not feel it should be discarded. It it is carried out with the care which Dr. Keene always olsserves in his work there is no danger. I have been delighted to hear the favorable trend of the discussion. This line of investigation typifies the accurate methods in many other branches of medicine, but only those who possess special skill should have charge of this class of cases. Even though the surgeon may be ever so skilful, he may not have the requisite training to arrive at proper conclusions if he attempts to apply this method himself, and, therefore, such cases should always be referred to men who through constant practice are especially skilled.

Dr. Henry K. Panconst, Philadelphia: Most of the remarks we have heard have been made from the point of view of the cystoscopist, and it might be well to say something from the roentgenologist's point of view. Three things are essential for correct diagnosis by this method: (1) injection ly one who thoroughly understands his work; (2) the making of the roentgenographic record; (3) correct interpretation of the findings. The roentgenologist is sometimes placed in an embarrassing position, as was recalled by Dr. Braasch's remark that the use of this method should be restricted to a few men. I feel thankful in having to do such work only in a hospital where the examinations are restricted to the services of men like Dr. Keene who know how to use the cystoscope and catheter. On one or two occasions I have seen some very poor work of this kind done. In his own office, at least, the roentgenologist should feel no hesitancy in refusing to examine cases for men who do not fully understand their part of the work.

\section{A REPORT OF TWENTY-SEVEN UNILAT- ERAL EXCLUSIONS OF THE PYLORIC REGION}

\section{WJTH SPECIAL REFFRENCE TO OPERATIVE TECHNIC *}

\author{
WILLARD BARTLETT, A.M., M.D. \\ ST. Lours"
}

It has been seven years since my first complete unilateral exclusion of the pyloric region was done by cutting tranversely across the stomach and closing the resulting ends blind. It seemed the only logical course of procedure when confronted by an enormons gastric ulcer situated a few centimeters from the pylorus and so extensively connected with the pancreas as to make resection seem inadvisable. The method proved itself valuable in several trials and naturally inspired an interest in its history. Doyen ${ }^{1}$ was formd to have been the first to record its performance in 1892, while von Eiselsberg ${ }^{2}$ reported two operations in 1895 without making mention of the French surgeon's work. For more than a decade following this time, the idea seems to have received little or no further attention until the present wave of enthusiasm for it started a very few years ago. In the accompanying table are reported my first fifteen cases in which the above-mentioned technic was used.

While the anatomic status obtained by complete transverse division of the stomach left nothing to be desired in fifteen cases, still I could not escape the impression that I had thereby added a very definite risk of shock, hemorrhage and sepsis to the risk attending the gastro-enterostomy as such. It was about this time that a surgical complication which befcll one of our ulcer cases demonstrated the possibility of functional exclusion of the pyloric region acting as at least a temporary substitute for the anatomic variety. It came about in this way.

Dec. 23, 1912, we resected most of the minor curvature in removing a large callous ulcer, thereby cutting the stomach more than half way in two. Careful transverse closure of the visceral wound was made, causing the pylorus to approach the cardia in the usual manner. witiout the lumen being encroached on; still a ccm-

\footnotetext{
* Read before the Section on Obstetrics, Gynecolugy and Abdomins Surgery at the Sixty-Fifth Annual Session of the American Medical Surgery at the Sixty-Fifth Annual Session
Associatin, Atlantic City, N. T., June, 1914. Association, Atlantic City, N. T., June, 1914.
1. Doyen: Französicher Chiro Kongress, 1893.

2. Von Eiselsberg: Arch. f. klin. Chir., 1895, L.
} 